\title{
Experimental evolution in Chlamydomonas. III. Evolution of specialist and generalist types in environments that vary in space and time
}

\author{
XAVIER REBOUD* \& GRAHAM BELL \\ Redpath Museum and Department of Biology, McGill University, 1205 Ave Dr Penfield, Montreal, Quebec, \\ Canada H3A 1B1
}

\begin{abstract}
Lines of Chlamydomonas were selected for growth either in Light or in Dark conditions for several hundred generations. Evolved lines that grew well in the environment of selection grew less well in the other environment, so that negative genetic correlation between Light and Dark growth was created by selection. The existence of a cost of adaptation was confirmed by reverse selection. The lines were also exposed to environments that varied either in space or in time with respect to Light and Dark conditions. Specialization (genetic variation) was retained in spatially variable environments, whereas generalization (phenotypic plasticity) evolved in temporally varying environments. The original negative correlation between adaptation to Light and Dark conditions seemed to be caused primarily by mutation accumulation rather than by antagonistic pleiotropy. It was thereby possible to select a generalist type nearly as well adapted in each environment as a specialist line.
\end{abstract}

Keywords: antagonistic pleiotropy, autotroph vs. heterotroph, Chlamydomonas, evolution of plasticity, mutation accumulation, selection experiment, trade-off.

\section{Introduction}

All environments vary to some degree in space and time; populations may respond by evolving a diverse array of specialists, or a single, highly plastic generalist. It is widely thought that adaptation to a specific environment is coupled to deterioration in certain other environments (see reviews by Futuyma \& Moreno, 1988; Sultan, 1992). This is conveniently expressed by treating performance in different environments as different traits (Falconer, 1952, 1981) that are negatively correlated among genotypes. This constitutes a 'cost of adaptation' that restricts the range of adaptation and underlies the evolution of specialization (see, for example, Levins, 1968; Roughgarden, 1972; Maynard Smith \& Hoekstra, 1980; Hedrick, 1986).

*Present address and correspondence: Institut National de la Recherche Agronomique, Laboratoire de Malherbologie, BV 1540, 21034 Dijon Cedex, France. E-mail: reboud@epoisses.inra.fr

(C) 1997 The Genetical Society of Great Britain.
Previous laboratory studies of the unicellular chlorophyte Chlamydomonas have shown that in arbitrary (unselected) populations the genetic correlation between environments differing in nutrient concentration is typically low, and falls towards zero as the difference between environments increases (Bell, 1991, 1992). When populations were cultured in heterogeneous environments, comprising a range of media differing quantitatively in macronutrient concentration, genetic variance of fitness was maintained, or at least eliminated more slowly than in a uniform environment (Bell, 1997). Moreover, clonal populations cultured in a heterogeneous environment that comprised two qualitatively different growth conditions, autotrophic growth in the light and heterotrophic growth in the dark, evolved substantial genetic diversity within a few hundred generations (Bell \& Reboud, 1997).

These experiments demonstrate that a cost of adaptation will contribute to the evolution of specialization and the maintenance of diversity. 
However, they do not address the issue of how a cost of adaptation arises.

\section{Antagonistic pleiotropy and mutation accumulation}

Genes which improve adaptation in both of two different environments are likely to become fixed more or less rapidly, whereas genes which reduce adaptation in both environments will be eliminated. Genes which improve adaptation in one environment while reducing adaptation in the other will be fixed or eliminated more slowly, and may even coexist indefinitely (see Levene, 1953; Via \& Lande, 1985, 1987; Gillespie \& Turelli, 1989). Such genes will, therefore, represent the bulk of genotypic variation observed in populations inhabiting a heterogeneous environment. The basis of the opposed fitness effects that they cause is a 'trade-off' arising from the allocation of finite resources to competing activities. Such trade-offs are often invoked to explain variation in characters such as gender allocation (reviewed by Charnov, 1982) and age-specific reproduction (reviewed by Reznick, 1985; Bell \& Koufopanou, 1986; Rose, 1991). The cost of adaptation is then one aspect of a general theory of antagonistic pleiotropy that interprets phenotypic evolution as leading to optimal states representing a compromise between opposed selection tendencies.

An alternative point of view is that the maintenance of any complex character requires the continual operation of natural selection. New variation will be created by mutation in every generation, but most of these mutations will be deleterious because any arbitrary change in a complex, welladapted character is likely to erode function (Fisher, 1930). Adaptation can therefore be maintained only if dysfunctional variants are continually removed by selection at the same rate at which they arise. Longcontinued exposure to one environment will reduce adaptation in certain other environments simply because adaptation in those other environments will decay through mutation for as long as it is not actively maintained by selection. The cost of adaptation then arises through the accumulation of mutations which are nearly neutral in the current environment of selection, but which are deleterious when expressed in certain other environments.

\section{Spatial and temporal variation}

If the environment varies in space, then both (or all) environments are simultaneously available. A lineage which is currently proliferating successfully in a given environment will be redistributed at inter- vals among different environments, but will also be reintroduced to the environment to which it is welladapted. Specialization can be maintained through antagonistic pleiotropy, because any given environment provides a refuge for types which thrive in it. In a clonal population, these specialized types will tend to accumulate mutations which are nearly neutral when expressed in the environment to which the type is adapted, although they are deleterious in other environments. (This would not be true in a sexual population, where genes that are deleterious in a given environment would be exposed to selection through being recombined with genes that are favoured in that environment.) The evolution of specialization in a spatially heterogeneous environment would thus be caused at first by antagonistic pleiotropy and later by mutation accumulation.

If the environment varies in time, then every lineage is compelled to grow first in one environment and then in another. It is very difficult to maintain specialized types through antagonistic pleiotropy in these circumstances, because one type or the other will have the greatest geometric mean rate of increase (Gillespie, 1977; Gavrilets \& Scheiner, 1993), and will therefore replace all its competitors. (This is not necessarily true in sexual diploids, where the type with the greatest geometric rate of increase in fitness may be a heterozygote; see Haldane \& Jayakar, 1963.) Mutation accumulation will be minimal, because mutations which are nearly neutral in one generation are likely to be deleterious in the altered conditions of growth experienced by the next generation. Environments which vary in time are therefore likely to favour plastic, generalist types.

Now, suppose that a variable environment is inoculated with a mixture of populations which have each become adapted to particular conditions of growth through long-continued selection, and express a cost of adaptation in terms of reduced growth in other conditions. If the new environment varies in space, the evolved specializations may be maintained provided that selection is sufficiently intense, and any cost of adaptation arising from antagonistic pleiotropy will continue to be expressed. The cost attributable to mutation accumulation will decline somewhat: although fitness is not affected by these mutations in the environment in which a given type has been selected, in any other environment individuals of that type with fewer mutations will increase in frequency relative to those with more mutations. However, this will be a weak effect if the production of any given type is dominated by the environment in which it was selected. If the new 
environment varies in time, then selection will favour generalist types which eventually replace the specialists. If specialization were based on antagonistic pleiotropy, then the generalists will be inferior in any environment to the original specialist. On the other hand, if a cost of adaptation were solely the outcome of mutation accumulation, then the generalist will evolve through the elimination of these mutations, and the generalist will be as fit in each environment as the original specialist.

We report here an experiment designed to test these ideas by selecting differently specialized populations in environments that vary in space or time. One of the most important features of our experiment is that the selection lines were originally founded by a single spore and were afterwards propagated vegetatively, so that all the variance and covariance they displayed arose through novel mutations during the course of the experiment. Our results do not depend therefore on the nature of the genetic variances and covariances pre-existing in the base population.

\section{Materials and methods}

\section{Concept of the experiment}

We used lines of Chlamydomonas reinhardtii that had been maintained asexually on minimal medium in the Light or on acetate-supplemented medium in the Dark, as a continuation of the experiment reported by Bell \& Reboud (1997). These base lines were transferred to each of four experimental treatments.

1 Continued selection. Light lines continued to be maintained in the Light environment, and Dark lines in the Dark environment. Provided that the base lines had each approached evolutionary equilibrium in the environment of selection, we expect little or no further response.

2 Reverse selection. Light lines were transferred to the Dark environment, and maintained there during the course of the experiment; similarly, Dark lines were transferred to the Light environment. Each line will then become adapted to the novel reverse environment to which it has been transferred. If the cost of adaptation displayed by the base lines, and by the continued lines, is attributable to antagonistic pleiotropy, adaptation will proceed by the substitution of genes which trade off increased adaptation to the reverse environment for decreased adaptation to their base environment. There will therefore be a continual increase in adaptation in the reverse environment, accompanied by a continual decrease in adaptation to the base environment, until complete adaptation to the novel environment has been achieved. On the other hand, if the cost of adaptation is caused by mutation accumulation, adaptation to the reverse environment will at first be achieved without any regress when tested in the base environment, because it will involve only the replacement of the conditionally deleterious mutations which accumulated during selection in the base environment. It is only subsequently that regress will develop, as mutations neutral in the reverse environment but deleterious in the base environment gradually accumulate through time.

3 Temporal selection. Lines were cultured alternately in the two environments. Thus, Light lines were transferred to the Dark environment for one cycle of growth, then back to Light, then to Dark again, and so forth. The theory of antagonistic pleiotropy predicts that adaptation to either environment will be traded off against regress in the other, so that temporal lines will be inferior to the specialized continued line in both environments. On the other hand, mutations which are deleterious in either environment will be selected against very effectively, because in every generation only a single environment is available. Conditionally deleterious mutations will not accumulate, and those which have accumulated in the base populations will tend to be eliminated. The theory of mutation accumulation therefore predicts that generalists will evolve which are as fit as the specialist continued line in each environment.

4 Spatial selection. The pair of Dark and Light base lines developed from the same founder were mixed, and this mixture used to inoculate Dark and Light environments. This process of mixture and redistribution was then repeated after each growth cycle. Because the appropriate environment is always available for the growth of specialized types, they will tend to persist, and the composition of the population will be roughly the same as that of the mixture of the two base lines.

\section{Base populations}

The base populations were two lines extracted from the experiment described by Bell \& Reboud (1997). The founder of each line was a single random spore extracted from a cross between two unrelated wild isolates (CC-1010 and CC-1952). At the beginning of the present experiment the lines had been maintained as asexual cultures in Light and Dark environments for 70 weeks, representing about 1200 generations of the Light line and about 750 of the 
Dark line of each pair. The Light line was propagated by inoculating a $1 \mathrm{~L}$ flask containing $300 \mathrm{~mL}$ of Bold's minimal medium with $0.1 \mathrm{~mL}$ of the culture from the previous cycle, then allowing the population to grow for 3-4 days under continuous fluorescent illumination while being bubbled with sterile air. The initial inoculum comprised about $6 \times 10^{4}$ cells, and the final population about $2 \times 10^{8}$ cells. The Dark line was propagated in exactly the same way, except that the medium was supplemented with $1.2 \mathrm{~g} \mathrm{~L}^{-1}$ sodium acetate, the flasks were wrapped with aluminium foil to exclude light, and the growth period was 7 days. Further details of how the base populations evolved from the founders are given by Bell \& Reboud (1997). Routine laboratory procedures are described by Harris (1989).

\section{Selection procedure}

The straightforward serial transfer used in the Light-Dark experiment was modified to create continued, reverse, temporal and spatial modes of selection, each new line being set up in duplicate. There were thus 2 base populations $\times 4$ selection modes $\times 2$ replicates $=16$ selection lines. They were maintained for 2 months, during which the continued Light lines went through 19 growth cycles (about 200 generations) and the reverse Dark lines (base line Light, transferred to Dark in this experiment) through eight growth cycles (about 90 generations), the other lines being intermediate between these two extremes.

\section{Assay procedure}

After selection, four random spores were isolated from each of the 16 lines, and also from the base populations, which had been stored on solid medium during the experiment. Each spore was assayed in both Light and Dark conditions by inoculating $20 \mathrm{~mL}$ of growth medium held in a $50 \mathrm{~mL}$ culture tube and measuring turbidity with a Bausch \& Lomb Spec-20 spectrophotometer at roughly daily intervals until growth was complete. The entire assay was blocked by replicating it in two growth cabinets, each laid out as a complete independently randomized block. It thus comprised 16 lines $\times 4$ spores/ line $\times 2$ assay environments $\times 2$ replicates $=256$ cultures from the selection lines. We also ran 2 lines $\times 4$ spores/line $\times 2$ assay environments $\times 3$ replicates $=48$ cultures from the base populations as checks.

This procedure gives complete growth-curves, from which the logistic parameters $r$ and $K$ can be estimated. We also analysed $P_{10}$, the observed turbidity after 10 days, as a simple, robust and model-free measure of growth. All three measures gave similar results; for simplicity, we report only $P_{10}$ in this paper. The selection and assay conditions are different (flasks and culture tubes), but growth in the two environments is positively correlated (Bell \& Reboud, 1997).

\section{Results}

\section{Response to selection}

The means and standard deviations of growth parameters are given in Table 1 , and an overall analysis of variance in Table 2. Block effects are small and nonsignificant at $P=0.05$, but the variance among spores is substantial. The overall model is highly significant; the lines became strongly differentiated according to the nature and extent of environmental variation, the outcome depending on the original population (Light or Dark) from which they were drawn. The effect of each treatment is shown in Fig. 1.

1 Base populations. These showed a strong negative genetic correlation caused by a cost of adaptation;

Table 1 Response to selection. Means and phenotypic standard deviations are calculated over two replicate cultures of each of four spores. 'Dark' and 'Light' selection environments for the spatial treatment refer to the condition of the last flask in the experiment

\begin{tabular}{|c|c|c|c|c|c|c|}
\hline \multirow[b]{3}{*}{ Treatment } & \multirow{3}{*}{$\begin{array}{c}\text { Selection } \\
\text { environment }\end{array}$} & \multirow[b]{3}{*}{ Rep. } & \multicolumn{4}{|c|}{ Assay environment } \\
\hline & & & \multicolumn{2}{|c|}{ Dark } & \multicolumn{2}{|c|}{ Light } \\
\hline & & & Mean & $\sigma_{\mathrm{P}}$ & Mean & $\sigma_{\mathrm{p}}$ \\
\hline \multirow[t]{2}{*}{ Continued } & Dark & 1 & 417 & 72 & 299 & 84 \\
\hline & & 2 & 423 & 83 & 304 & 80 \\
\hline \multirow[t]{2}{*}{ Continued } & Light & 1 & 16 & 15 & 547 & 85 \\
\hline & & 2 & 9 & 5 & 603 & 129 \\
\hline \multirow[t]{2}{*}{ Reverse } & Dark & 1 & 327 & 145 & 713 & 34 \\
\hline & & 2 & 644 & 19 & 729 & 37 \\
\hline \multirow[t]{2}{*}{ Reverse } & Light & 1 & 36 & 26 & 588 & 67 \\
\hline & & 2 & 329 & 193 & 455 & 74 \\
\hline \multirow[t]{2}{*}{ Temporal } & Dark & 1 & 334 & 109 & 454 & 211 \\
\hline & & 2 & 454 & 128 & 422 & 180 \\
\hline \multirow[t]{2}{*}{ Temporal } & Light & 1 & 455 & 49 & 514 & 80 \\
\hline & & 2 & 464 & 77 & 519 & 83 \\
\hline \multirow[t]{2}{*}{ Spatial } & (Dark) & 1 & 160 & 174 & 435 & 216 \\
\hline & & 2 & 270 & 198 & 497 & 189 \\
\hline \multirow[t]{2}{*}{ Spatial } & (Light) & 1 & 64 & 89 & 647 & 85 \\
\hline & & 2 & 48 & 80 & 607 & 140 \\
\hline
\end{tabular}

(C) The Genetical Society of Great Britain, Heredity, 78, 507-514. 
Table 2 Overall analysis of variance for the experiment. Blocks are two replicate assays. Residual variance confounds variance among replicate cultures with replicate $\times$ block interaction. Variance of spores confounds variance among spores within lines with variance among replicate selection lines. The spores mean square was used to test model effects. These are: origin (Light or Dark), selection treatment (continued, reverse, temporal or spatial), and their interaction

\begin{tabular}{lrrc}
\hline & & \multicolumn{2}{c}{ Mean squares } \\
\cline { 3 - 4 } Source & df & \multicolumn{1}{c}{ Dark } & Light \\
\hline Block & 1 & $8482 \mathrm{NS}$ & $5202 \mathrm{NS}$ \\
Model & 7 & $554971^{* * *}$ & $256693^{* * *}$ \\
$\quad$ Origin & 1 & $1294038^{* * *}$ & $196251^{*}$ \\
Selection treatment & 3 & $527442^{* * *}$ & $207837^{* * *}$ \\
$\quad$ Origin $\times$ Selection & 3 & $336145^{* * *}$ & $325697^{* * *}$ \\
Spores & 56 & $33127^{* * *}$ & $27842^{* * *}$ \\
Residual & 63 & 8380 & 8549 \\
\hline
\end{tabular}

${ }^{*} P \leqslant 0.05,{ }^{* * *} P \leqslant 0.001$

the Dark lines grew well in the Dark environment and poorly in the Light, whereas the Light lines grew well in the Light but were almost unable to grow in the Dark. This confirms the observations of Bell \& Reboud (1997).

2 Continued selection. This pattern was maintained, as expected, in the continued lines, without any evident enhancement of specialization.

3 Reverse selection. Under reverse selection, lines from the Dark base population rapidly improved, to reach levels of performance in the Light comparable with those of the continued Light lines. The reverse lines from the Light base population were heterogeneous; some spores had evolved the ability to grow well in the Dark, whereas others showed little or no improvement. The expression of a cost of adaptation within these lines was variable. Most spores from the lines switched from Dark to Light grew better in the Light with no loss of ability to grow in the Dark. Some spores from one of the two replicate lines grew much less well in the Dark, but this was not associated with superior growth in the Light. On the other hand, the spores from lines switched from Light to Dark environments which grew well in the Dark grew slightly less well in the Light.

4 Temporal selection. Under temporal selection, most spores showed an increased ability to grow in the novel environment, although some from the
Dark base population showed no improvement in the Light. No negative genetic correlation is apparent in these results.

5 Spatial selection. By contrast, specialization seems to have been retained under spatial selection. The tight clustering of specialized genotypes in the continued lines breaks down to some extent, but an overall negative genetic correlation is retained.

\section{The cost of adaptation in variable environments}

By expressing the mean performance of spores from different selection treatments as deviations from the continued lines, the extent of advance and regress in the two environments can be seen more clearly (Fig. 2). Reverse or temporal selection applied to the Light base population caused a substantial improvement in Dark growth, with an appreciable but lesser regress in Light growth. Similarly, reverse selection applied to the Dark base population caused substantial advance in the Light and a modest regress in the Dark, although temporal selection had little effect. The spatial lines, regardless of their origin, deteriorated slightly in the novel environment, without improving in the environment in which their base population had been selected.

\section{Discussion}

\section{Response to selection}

The continued lines show that selection applied for about a thousand generations had been sufficient to bring the base populations close to evolutionary equilibrium, in the sense that little adaptive change occurred at the scale of the hundred or so generations occupied by the experiment. There is, nevertheless, the potential for rapid evolutionary change in these large asexual populations on this time-scale, demonstrated by the general improvement of the reverse lines.

Selection in Light and Dark environments is not necessarily symmetrical. Even after long-continued selection, lines maintained in the Dark retain the capacity to grow reasonably well in the Light, whereas lines maintained in the Light become at length almost unable to grow at all in the Dark. All the spores assayed from the reverse lines switched from Dark to Light could grow as well in the Light as those from the continued Light lines; selection over a hundred generations was as effective as selection over a thousand generations. By contrast, only a minority of spores from lines that were switched from Light to Dark grew well in the Dark, and most 

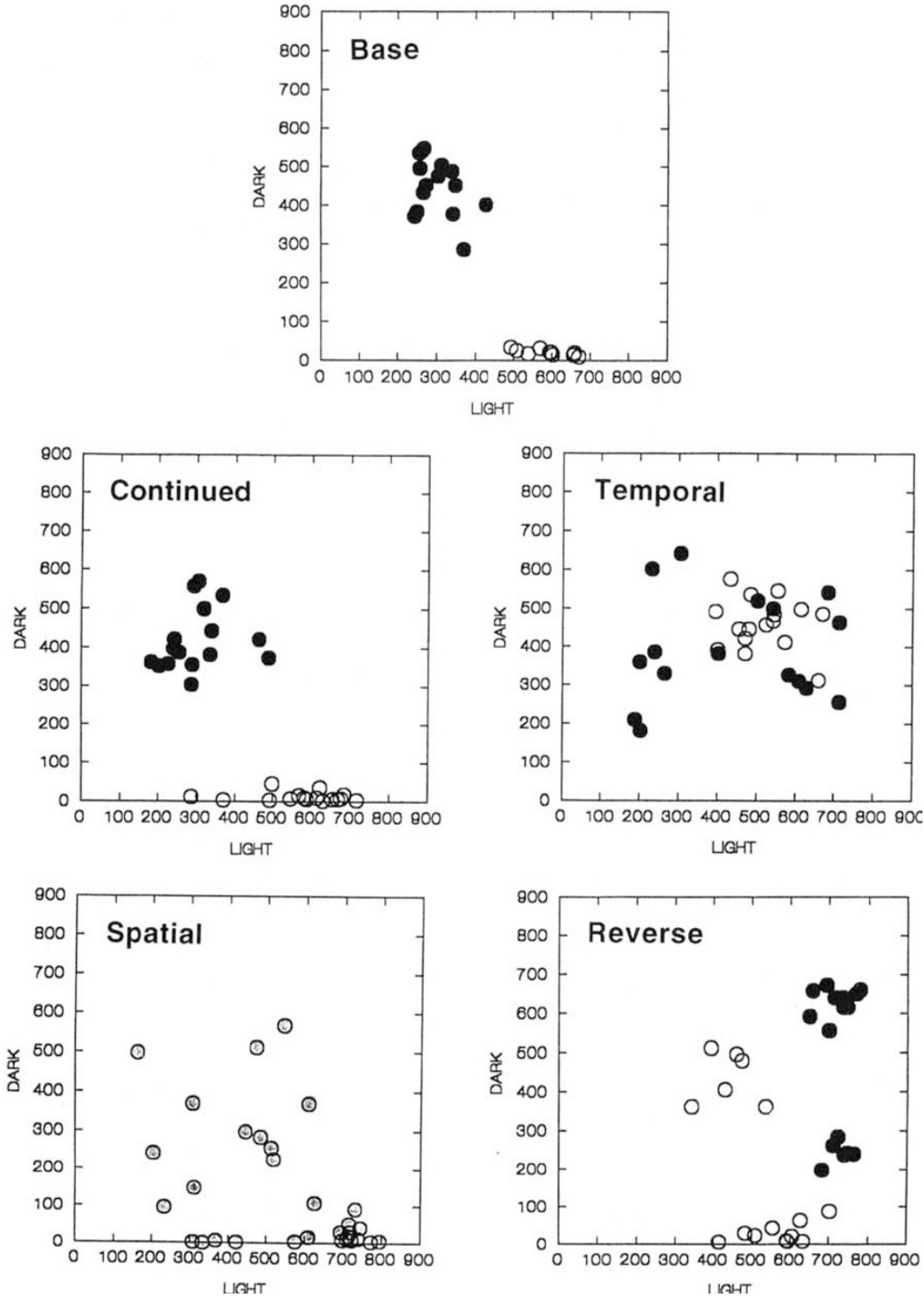

Fig. 1 Response to selection in the experimental lines. Solid circles indicate selection in the Dark, open circles in the Light. Each plotted point is the value of $P_{10}$ for a culture. showed little if any improvement. This reflects the fact that one line had responded to selection whereas the other had not.

\section{Cost of adaptation}

We have previously demonstrated a cost of adaptation in the base populations by comparing the selection lines with their founders: improvement in the environment of selection was associated with regress in the other environment (Bell \& Reboud, 1997). This result was confirmed in the present experiment by the behaviour of the reverse lines, which tended to lose part of the adaptation they had previously evolved. The indirect response to selection in a novel environment was thus generally antagonistic.

\section{Maintenance of diversity}

In our previous experiment, lines founded by a single spore evolved relatively high levels of genetic variance when maintained under spatial heterogeneity for several hundred generations. These spatial lines were inferior to sister lines that had been maintained exclusively in one of the two environments, when tested in that environment, but were generally superior in the other environment. Here, we describe the converse procedure of first 


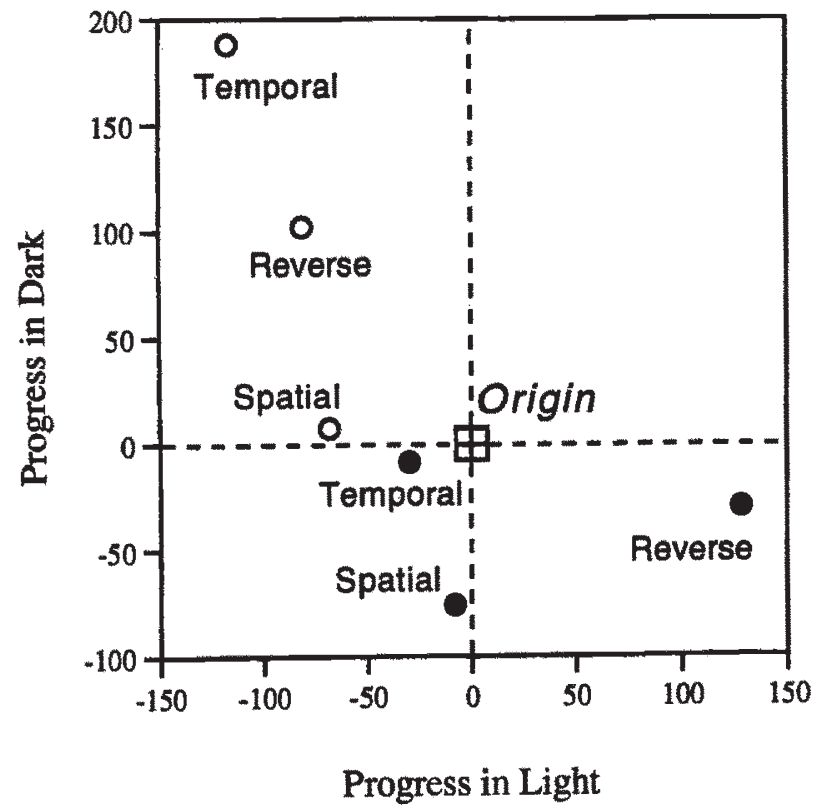

Fig. 2 Response to selection relative to the continued lines. Solid circles indicate selection in the Dark, open circles in the Light. Plotted points are treatment means based on two replicates of four spores in each of two replicate selection lines.

evolving specialized lines in isolation, then mixing the divergently specialized lines descending from the same spore, and propagating this mixture in a spatially heterogeneous environment. This caused slight regress in both environments, as expected. At the same time, there was no substantial loss of genetic variance from this mixed population during about a hundred generations of selection. We have therefore demonstrated that fitness variance evolves in genetically uniform populations and is maintained in genetically diverse populations through selection in a spatially heterogeneous environment, primarily as the consequence of a cost of adaptation.

\section{Evolution of plasticity}

The temporal lines evolved greatly enhanced phenotypic plasticity. Indeed, the most striking result of the experiment was the appearance in temporal lines of spores able to grow in either environment as successfully as the specialists from the continued lines. Our results give experimental support to the view that specialization evolves in environments that vary in space, whereas generalization evolves in environments that vary through time. The outcome of selection in natural populations is likely to depend, not only on the extent of environmental variation, but also on the balance between spatial and temporal variation.

\section{Antagonistic pleiotropy and mutation accumulation}

The evolution of superior generalists in the temporal lines may seem unexpected, because it appears to contradict the cost of adaptation expressed by the other lines in the experiment. However, the evolution of generalization in environments that vary through time depends on the cause of the cost of adaptation.

The reverse lines express a cost of adaptation that may be caused by antagonistic pleiotropy, because it arises within the time-span of the experiment. The reverse lines extracted from the Light base population and subsequently selected in the Dark still contained many spores that were scarcely able to grow in the Dark. They also contained a substantial minority of spores that grew well in the Dark but grew somewhat less well in the Light. This regress was much less, however, than the regress observed in the base and continued Dark lines. Over the course of the experiment therefore only a fraction of the eventual cost of adaptation was expressed. A similar pattern was found in the Dark base lines transferred to the Light, although in this case regress in the original environment was even less pronounced. We conclude that antagonistic pleiotropy made a detectable but minor contribution to the cost of adaptation.

The evolution of superior generalists in the temporal lines is very difficult to understand, if antagonistic pleiotropy were the main cause of the cost of adaptation. However, it is consistent with the theory of mutation accumulation, given that the experiment was too brief to permit a substantial load of conditionally deleterious mutations to accumulate. It is also consistent with the rather slight regress observed in the reverse lines. We conclude that mutation accumulation makes the major contribution to the cost of adaptation in Light and Dark environments.

\section{The effect of long-continued selection}

It seems likely that the average difference between the effects of a mutation in two environments will vary directly with the ecological difference between them. Most mutations that are neutral in a given environment will be nearly neutral in a similar environment, whereas many will be markedly deleterious in a very different environment. In other words, the probability that a mutation, neutral in 
one environment, will be markedly deleterious in another will increase with the ecological difference between them. Nevertheless, mutations may occasionally arise that have deleterious effects even in a very similar environment, and these will accumulate very slowly. If this argument is correct, then even very similar environments will generate a cost of adaptation, after a sufficiently long period of time. The evolution of specialization then depends, not only on the difference between environments, but also on the length of time over which lines are selected in these environments.

\section{Acknowledgements}

This work was funded by a research grant from the Natural Sciences and Engineering Research Council of Canada. X.R. was supported by a Postdoctoral Fellowship from the Ministere de la Recherche et de la Technologie, France. We are grateful to Lori Pilkonis for technical assistance.

\section{References}

BELL, G. 1991. The ecology and genetics of fitness in Chlamydomonas. III. Genotype-by-environment interaction within strains. Evolution, 45, 668-679.

BELL, G. 1992. The ecology and genetics of fitness in Chlamydomonas. V. The relationship between genetic correlation and environmental variance. Evolution, 46, $561-566$.

BELL, G. A. C. 1997. Experimental evolution in Chlamydomonas. I. Short-term selection in uniform and diverse environments. Heredity, 78, 490-497.

BELL, G. AND KOUfOPANOU, v. 1986. The cost of reproduction. In: Dawkins, R. \& Ridley, M. (eds) Oxford Surveys in Evolutionary Biology, vol. 3, pp. 83-131. Oxford University Press, Oxford.

BELL, G. AND REBOUD, X. 1997. Experimental evolution in Chlamydomonas. II. Genetic variation in strongly contrasted environments. Heredity, 78, 498-506.

Charnov, E. L. 1982. The Theory of Sex Allocation. Princeton University Press, Princeton, NJ.
FALCONER, D. S. 1952. The problem of environment and selection. Am. Nat., 86, 293-298.

FALCONER, D. S. 1981. Introduction to Quantitative Genetics, 2nd edn. Longman, Harlow, Essex.

FISHER, R. A. 1930. The Genetical Theory of Natural Selection. Clarendon Press, Oxford.

FUTUYMA, D. J., AND MORENO, G. 1988. The evolution of ecological specialization. Ann. Rev. Ecol. Syst., 19, 133-143.

GAVRILETS, S. AND AND SCHEINER, S. M. 1993. The genetics of phenotypic plasticity. V. Evolution of reaction norm shape. J. Evol. Biol., 6, 31-48.

GILlESPIE, J. H. 1977. Natural selection for variance in offspring numbers: a new evolutionary principle. $\mathrm{Am}$. Nat., 111, 1010-1014.

GILlESPIE, J. H. AND TURELLI, M. 1989. Genotype-environment interactions and the maintenance of polygenic variation. Genetics, 121, 129-138.

HAlDANE, J. B. S. AND JAYAKAR, S. D. 1963. Polymorphism due to selection of varying direction. J. Genet., 58, 237-242.

HARRIS, E. 1989. The Chlamydomonas Source-Book. Academic Press, New York.

HEDRICK, P. W. 1986. Genetic polymorphism in heterogeneous environments: a decade later. Ann. Rev. Ecol. Syst., 17, 535-566.

LEVENE, H. 1953. Genetic equilibrium when more than one ecological niche is available. Am. Nat., 78, 331-333.

LEVINS, R. 1968. Evolution in Changing Environments. Princeton University Press, Princeton, NJ.

MAYNARD SMITH, J. AND HOEKSTRA, R. 1980. Polymorphism in a varied environment: how robust are the models? Genet. Res., 35, 45-57.

REZNICK, D. 1985. Costs of reproduction: an evaluation of the empirical evidence. Oikos, 44, 257-267.

ROSE, M. R. 1991. Evolutionary Biology of Aging. Oxford University Press, Oxford.

Roughgarden, J. 1972. Evolution of niche width. Am. Nat., 106, 683-718.

sultan, s. E. 1992. Phenotypic plasticity and the Neo-Darwinian legacy. Evol. Trends Plants, 6, 61-68.

VIA, S. AND LANDE, R. 1985. Genotype-environment interaction and the evolution of phenotypic plasticity. Evolution, 39, 505-522.

VIA, S. AND LANDE, R. 1987. Evolution of genetic variability in a spatially heterogeneous environment: effects of genotype-environment interaction. Genet. Res., 49, 147-156. 